\title{
Universal community nucleic acid testing for COVID-19 in Hong Kong reveals insights into transmission dynamics
}

\section{Bingyi Yang}

The University of Hong Kong

Tim K. Tsang

The University of Hong Kong

Huizhi Gao

The University of Hong Kong

Eric H. Y. Lau

The University of Hong Kong

Yun Lin

The University of Hong Kong

\section{Faith Ho}

The University of Hong Kong

Jingyi Xiao

The University of Hong Kong

Jessica Y. Wong

The University of Hong Kong

Dillon C. Adam

The University of Hong Kong

Qiuyan Liao

The University of Hong Kong

Peng Wu ( $\sim$ pengwu@hku.hk)

The University of Hong Kong

Benjamin J. Cowling

The University of Hong Kong

Gabriel M. Leung

The University of Hong Kong

\section{Research Article}

Keywords: Mass testing for COVID-19 
Posted Date: May 20th, 2021

DOI: https://doi.org/10.21203/rs.3.rs-542072/v1

License: (c) (i) This work is licensed under a Creative Commons Attribution 4.0 International License. Read Full License 


\section{Abstract}

Background: Testing of an entire community has been used as an approach to control COVID-19. In Hong Kong, a universal community testing programme (UCTP) was implemented at the fadeout phase of a community epidemic in July to September 2020, to determine the prevalence of unrecognised cases and limit any remaining transmission chains. We described the utility of the UCTP in finding unrecognised cases, and analysed data from the UCTP and other sources to characterise transmission dynamics.

Methods: We described the characteristics of people participating in the UCTP, and compared the clinical and epidemiological characteristics of COVID-19 cases detected by the UCTP versus those detected by clinical diagnosis and public health surveillance. We developed a Bayesian model to estimate the agespecific incidence of infection and the proportion of cases detected by clinical diagnosis and public health surveillance.

Findings: 1.77 million people, $24 \%$ of the Hong Kong population, participated in the UCTP from 1 to 14 September 2020. The UCTP identified 32 new infections (1.8 per 100,000 samples tested), consisting of $29 \%$ of all local cases reported during the two-week UCTP period. Compared with the existing clinical diagnosis and public health surveillance, the UCTP detected a higher proportion of sporadic cases (62\% versus $27 \%, \mathrm{p}<0.01$ ) and identified 6 (out of 18 ) additional transmission chains during that period. We estimated that $27 \%$ (95\% credible interval: $22 \%, 34 \%$ ) of all infections were detected by the existing clinical diagnosis and public health surveillance in the third wave.

Interpretation: We reported empirical evidence of the utility of population-wide COVID-19 testing in detecting unrecognised infections and transmission chains. Around three quarters of infections have not been identified through existing surveillance approaches including contact tracing.

\section{Introduction}

Hong Kong is a subtropical city on the southern coast of China with a population of 7.5 million. A number of surges in coronavirus disease 2019 (COVID-19) transmission have been successfully suppressed through effective public health and social measures (PHSMs), and as of 6 May 2021 there have been a total of 11,797 laboratory-confirmed cases among which 210 have been fatal. ${ }^{1}$ Cases are detected through clinical and public health surveillance, targeting persons with acute respiratory symptoms, close contacts of confirmed cases, and high-risk groups (Table S1). ${ }^{2}$ Since the beginning of the pandemic, all confirmed cases including asymptomatic cases have been isolated in hospital until symptom resolution and either two consecutively negative PCR tests at least 24 hours apart or a positive ELISA IgG test; and the close contacts of confirmed cases have been traced and placed in strict quarantine for 14 days after their last exposure. There has been almost universal wearing of face masks outside the home, and intermittent use of social distancing measures including restricting public gatherings, requiring restaurants to operate at reduced capacity and for shorter hours, closing bars, nightclubs and karaoke centers, allowing civil servants to work from home, and suspending in-person schooling. ${ }^{3-5}$ 
While stay-at-home orders and universal testing have been a key feature of the response to surges in COVID-19 transmission in mainland China, ${ }^{6-8}$ Hong Kong has only once attempted a universal community testing program (UCTP) without a stay-at-home order for all Hong Kong residents aged 6 years or older who had not undergone throat or nasal operations. ${ }^{9}$ Respiratory samples were collected for testing by the reverse transcription polymerase chain reaction (RT-PCR). In Hong Kong's third epidemic wave of COVID-19, daily case numbers had surged to a peak of around 100 cases per day in June/July before declining to a low level by the end of August 2020. ${ }^{1}$ The objective of the UCTP in September 2020 was to identify unrecognised cases and transmission chains in the community in the third wave and thereby hasten the decline in daily case numbers down to zero. In total, 1.7 million (24\%) of Hong Kong residents were tested. Here we describe the results of the UCTP and draw broader inferences on the transmission dynamics of COVID-19 and the utility of the UCTP in Hong Kong.

\section{Materials And Methods}

\section{Sources of data}

A detailed line list of laboratory-confirmed cases of COVID-19 was obtained from the Department of Health of the Government of the Hong Kong Special Administrative Region, supplemented with clinical data from the Hospital Authority which operates the public hospital system and manages all confirmed cases in Hong Kong. Regarding the UCTP we obtained data on the number of tests completed and positives that were aggregated by district, sex and age group from the Department of Health.

We conducted a cross-sectional telephone survey between 31 August to 4 September to assess the responses to the UCTP among Hong Kong adult residents $(n=508)$, which is part of a series of telephone surveys described elsewhere. ${ }^{3,10}$ Study participants were sampled from random digit dialling with a 1:1 ratio of landline and mobile numbers. Among a variety of data collected on attitudes and behaviours in response to COVID-19, we collected data on intention to participate in the UCTP and concerns about the UCTP in the survey.

\section{Statistical analysis}

We performed a descriptive analysis on the testing results of the UCTP. The proportion of test positives in the UCTP was calculated by dividing the number of new positive samples by the number of total tests completed. The $95 \%$ confidence intervals $(\mathrm{Cl})$ were derived assuming a binomial distribution. Chi-squared tests and Fisher exact tests were used to compare the differences in positive proportions by sex and age group.

Hong Kong is divided into 18 geographic districts. We calculated the district- and age-specific participation proportions as the number of completed tests over the total population in each district or age group. Data on district- and age-specific population were obtained from the 2016 Population By- 
census in Hong Kong. ${ }^{11}$ We compared the participation proportions with the preceding incidence, which was calculated as the number of laboratory-confirmed locally infected COVID-19 cases between 1 July and 31 August 2020 (i.e., the two months preceding the UCTP) divided by the population in each district or age group. In the telephone survey data, we calculated age-specific proportions of intended participation as the number of survey responders who indicated certain, very likely or likely in attendance of the UCTP divided by the total number of respondents in each age group. The proportions expressing concern were calculated as the number of responders that expressed a specific concern about the UCTP divided by the total number of respondents in pre-defined groups. We compared intended participation with actual participation proportions.

COVID-19 cases in Hong Kong have been detected through clinical diagnosis by testing inpatients and outpatients with respiratory illnesses, as well as public health surveillance involving testing of persons at potentially higher risk of infection including contacts of known cases and arriving travellers, persons with a relatively higher occupational risk of infection, and residents in heavily affected buildings or communities (Table S1). During the third pandemic wave (i.e., our study period, 1 July to 30 September 2020), high risk occupations were further expanded to include drivers, fishermen, and people working at supermarkets and post and container terminals (Table S1). ${ }^{9}$ In this study, we focused on persons infected in Hong Kong, and did not include imported cases. To compare the case detections from the UCTP with these existing approaches, we obtained data on laboratory-confirmed local COVID-19 cases from the Department of Health, ${ }^{2}$ including the information on age, report date, symptomatic status and onset date, cycle threshold $\left(C_{t}\right)$ values for the confirmation test, dates and length of hospital stay, and epidemiological history. A cluster was defined as two or more confirmed cases were epidemiologically linked. ${ }^{12}$ Cases that had the earliest symptom onset time within a cluster or who were not linked to any other cluster were defined as "possible primary" cases, ${ }^{12}$ otherwise, were defined as "possible contacts". We used chi-squared tests to compare the differences in demographics and a Mann-Whitney $U$ test to compare differences in $\mathrm{C}_{t}$ values between cases detected from the UCTP and from the clinical diagnosis and public health surveillance. We used Wald test under Cox regression model to compare differences in length of hospital stay adjusting for age between the cases detected from the two sources.

We fitted a Bayesian model to data on age-specific death counts and the time series of cases reported through the clinical diagnosis and public health surveillance during the third wave and the UCTP to jointly estimate the age-specific proportion of detection by the existing surveillance after infections and the epidemic growth rate with prior information on the age-specific infection-fatality risk (IFR) (Table S2). Data on age-specific deaths were retrieved in late January 2021 when all the cases from the third wave had resolved. In this model, for 
age group $j$, the daily incidence of infections exponentially increased following a rate $\left(r_{1, j}\right)$ before changing on the date $\left(t_{c}\right)$, and then exponentially decreased with a rate $\left(r_{2, j}\right)$ for each age group $j$, to reflect the impact of the implemented PHSMs on the control of the third wave. Then, an age-dependent fraction of infected individuals $\left(\lambda_{j}\right)$ was detected and tested by the existing clinical diagnosis and public health surveillance. During the period of the UCTP, we assumed all infections among people participating in the UCTP (participation proportion, $p_{j}$ ) were detected. Together with the delay distribution from infection to report (estimated from the Hospital Authority data and a previous study ${ }^{13}$; Figure S1) and time-varying test sensitivity, ${ }^{14}$ we computed the model-expected age-specific daily numbers of reported cases detected by the existing surveillance and the UCTP (see Appendix for details). We also calculated the total number of deaths during 1 July to 30 September using the total number of infections and the standardised IFR for each age group (Table S3 $)^{15}$. Hence, we fitted the model to the data by linking the model-expected daily number of reported cases with the observed one by Poisson distributions. The same was also performed for the modelexpected and observed total number of deaths (see Appendix for details). Parameters were estimated by Metropolis-Hastings Monte Carlo Markov chain algorithm. We examined four age groups (i.e., 6-19, 20-64, 65-79 and 80), as the UCTP did not target children younger than 6 years of age and a substantially higher IFR was suggested among older adults. ${ }^{15}$

\section{Ethical approval}

Our project was approved by the Institutional Review Board of the University of Hong Kong/Hospital Authority Hong Kong West Cluster (HKU/HA HKW IRB).

\section{Results}

The UCTP was conducted between 1 to 14 September 2020 in 148 test centres across all 18 districts of Hong Kong (Figure 1A). In total, 1,783,232 samples were tested from 1,765,549 individuals ( $24 \%$ of the total population), including a very small number of repeated samples (1\%) from the same individuals (Table S4). Age and sex were available for $99.8 \%$ of the samples, of which $70.0 \%$ were aged between 20 to 64 years old (Table 1 ).

The district- and age- specific participation proportions were broadly consistent with the corresponding preceding incidence of confirmed COVID-19 cases (Figure 1). The districts of Wan Chai and Central and Western had the highest participation proportion (39\% and 32\%, respectively) but lower preceding incidence compared to the other districts (Figure 1B). Although adults aged 20 to 29 had a high preceding incidence, their participation proportions ( $8.9 \%$ and $11.0 \%$ respectively) were the lowest among all eligible age groups (Figure 1C). Consistently, we found relatively lower intention to participate $(26.2 \%, 95 \% \mathrm{Cl}$ : 
$20.1 \%, 33.0 \%$ ) and a higher proportion of concerns about participation in the UCTP in adults 18 to 39 years of age compared to other age groups in the telephone survey (Figure 2).

The UCTP identified a total of 40 individuals with a positive RT-PCR result. Among them, 32 were newly identified COVID-19 cases, and 8 were recovered individuals from previously confirmed COVID-19 who were determined by public health officers to have long-term virus shedding rather than re-infections. All these 8 recovered cases with long-term shedding, comprising $1.3 \%(95 \% \mathrm{Cl}: 0.6 \%, 2.6 \%)$ of 611 recovered were tested in the UCTP, were not thought to be infectious at the time of testing as they were discharged from hospital within the preceding month following complete symptom resolution and had detectable IgG antibodies. As such, these 8 cases were not included in further analyses.

The 32 newly-identified cases represented $29 \%$ (95\% Cl: $21 \%, 38 \%)$ of the 111 local cases that were identified during the two-week UCTP program (Figure 3A). The proportion of test positives of the UCTP was 1.8 (95\% Cl: $1.2,2.5)$ per 100,000 samples tested, with the highest proportions among people aged over 80 years of age $(6.6,95 \% \mathrm{Cl}: 1.8,16.8$ per 100,000 ; Table 1$)$. Ct values were similar between cases detected in the UCTP and those from the clinical diagnosis and public health surveillance (geometric mean 27.2 vs. 25.0, Mann-Whitney U test, $p=0.26$ ) (Figure 4A).

Among the 32 newly identified cases, $41 \%(n=13)$ had no symptoms at the time of testing, which was similar to that $(29 \%, 23$ out of 79 ; chi-squared test, $p=0.34)$ among cases detected from existing surveillance during the UCTP period (Figure 3). Of note, 4 cases developed symptoms on or after the day of the test. The median length of hospital stay was similar between cases detected by the UCTP and those detected from existing surveillance in September ( 9.5 vs. 11 days, Wald test under a Cox regression model, $p=0.77$ ) after adjusting for age (Figure 4B).

About $62 \%(n=20)$ of the newly identified cases from the UCTP had no epidemiological link with any other confirmed COVID-19 cases as their potential infectors, which was higher than those $(27 \%, 21$ out of 79; chi-squared test, $p<0.001$ ) detected from the clinical diagnosis and public health surveillance during the same period. Among these 20 possible primary cases, $70 \%(n=14)$ did not appear to transmit to any individual. Two of them (out of 20,10\%) transmitted to three of their household members who were also detected in the UCTP, and four (20\%) transmitted to household members who were later detected through contact tracing (Figure 3B). These six transmission chains consisted of $33 \%(n=18)$ of the newly identified transmission chains during the UCTP period. Nine of the newly confirmed cases were found to have epidemiological links to two known clusters identified by the existing surveillance, and five were contacts of the possible primary cases of the clusters, suggesting at least $35.7 \%$ (95\% Cl: $12.8 \%, 64.9 \%$ ) of the possible secondary cases in the two clusters might have been missed by contact tracing (Figure 3B).

By comparing cases identified through the UCTP and the existing clinical diagnosis and public health surveillance, we were able to estimate the proportion of all infections that were being detected by the existing surveillance. In our Bayesian modelling analysis, we estimated that the incidence of infections during the third wave was 1.3 (95\% credible interval (Crl): $0.6,2.8)$ per 1,000 for individuals 6 to 19 years 
old, 1.5 (95\% Crl: 1.2, 2.0) per 1,000 for aged 20 to $64,1.9$ (95\% Crl: 1.4, 2.5) per 1,000 for 65 to 79 and 2.5 $(95 \% \mathrm{Crl}: 2.0,3.2)$ per 1,000 for those at 80 years of old or above (Figure $5 \mathrm{~A})$. We estimated a total of $12,000$ (95\% Crl: $9,800,15,000)$ infections occurred in people aged above 6 years in the third wave in Hong Kong, suggesting an overall $27 \%$ (95\% Crl: $22 \%, 34 \%)$ of all infections were detected by existing surveillance during that period. The highest proportion of detection ( $56 \%, 95 \% \mathrm{Crl}: 50 \%, 61 \%)$ was in persons aged 20 to 64 years old (Figure 5B), who were also more likely to be detected as "possible primary" cases compared to other age groups (Table S5).

\section{Discussion}

In this study, we reported the experience of population-wide mass testing in Hong Kong that was performed in the fadeout phase of the third pandemic wave in Hong Kong. Around $25 \%$ of the eligible people participated in the UCTP. The low proportion of test positives (1.8 per 100,000 samples) in the UCTP indicated a low prevalence of COVID-19 in the community at the time of the UCTP. However, results from the reconstructed transmission network suggested that the UCTP still captured several hidden transmission chains that were missed by the existing clinical diagnosis and public health surveillance. We estimated that the exiting surveillance captured on average $27 \%$ (95\% Crl: $22 \%, 34 \%$ ) of the total infections in the third wave, with the highest identification in people aged 20 to 64 years old possibly due to higher detections of cases from the sources other than contact tracing in this age group. The incidence of infections during the third wave was estimated to increase with age, indicating that testing and surveillance should be enhanced for older adults. Our results confirmed the utility of mass testing in identifying unrecognised cases and transmission chains in the community with a relatively low risk of infection, although we did not quantify the cost-effectiveness of the UCTP.

Testing more than 1.7 million persons in the UCTP allowed health authorities to identify six (out of 18) additional transmission chains that might have been missed by the existing surveillance, and prevented onward transmission in the community, although the probability of these additional cases further causing superspreading events may not be very high, given the transmission heterogeneities and the strict PHSMs implemented in Hong Kong during the time. ${ }^{12}$ We also found several contacts of the existing clusters in the existing surveillance that appeared to have earlier isolation compared to the other contacts in those clusters who were detected through contact tracing (Figure S3). Such findings provided empirical evidence that population-wide mass testing could reduce potential onward transmission on top of proactive symptomatic case detection and contact tracing. ${ }^{16,17}$ In addition, our results confirmed that there have been unrecognised cases and community transmission in the fadeout phase of an outbreak, suggesting the potential risk of resurgence if PHSMs are relaxed too early.

While persons with symptoms were not eligible to participate in the UCTP, and instead should have sought medical attention and been tested through public or private outpatient clinics, there were a number of symptomatic cases identified through the UCTP. Testing under the UCTP was provided for free, while at the time there was a consultation fee associated with testing in outpatient clinics. Following the 
UCTP, 21 community testing centres were launched to provide free testing for certain pre-defined risk groups and self-paid voluntary testing for the general population.

We found that the proportion of test positives in the UCTP and the inferred incidence of COVID-19 infections generally increased with age in Hong Kong, which was consistent with findings from the mass testing exercise in Wuhan. ${ }^{6}$ Meanwhile, a series of assessments of community infections in the United Kingdom found that persons over 65 years of age might have a lower risk of infection, compared to other age groups. ${ }^{18}$ The age distributions of infections could be simultaneously affected by the socioeconomic status of the population and PHSMs that were implemented to control the pandemic. For our findings in particular, the low incidence of infections among children could be partially due to the school holidays and school suspensions between mid-July to mid-September, ${ }^{9}$ which could have reduced the risk of COVID-19 transmission in children. ${ }^{19-21}$

Our findings also suggested that people aged 6 to 19 years old and those 80 years or older were less likely to be picked up by the existing surveillance if infected but not linked with any other known cases (Table S5). During the study period, cases with no known epidemiological links were often detected through test for outpatients with respiratory symptoms at clinics or screening for inpatients at admission, therefore children could be missed due to their relatively milder symptoms if infected. Our findings suggested the importance of enforcing public health measures (e.g., testing and vaccination) for older adults and their caregivers, given the higher risk of infection and severe outcomes in infected older adults. $^{15}$

The Hong Kong UCTP is one of the few population-wide mass testing programmes of COVID-19 that have been conducted in China during a period of low incidence. The others were launched swiftly in responses to acute outbreaks except for Wuhan where mass testing was implemented after an extended period of lockdown. ${ }^{6-8}$ These mass testing programmes were often conducted for one or more rounds and targeted residents of entire cities, while in Shanghai, residents from affected areas were tested during outbreaks in November 2020. ${ }^{6-8}$ We found a lower proportion of test positives than that in Wuhan (3.0 per 100,000 , though $63 \%$ of positive cases had IgG antibody detected and none was infectious) and Beijing in June (3.1 per 100,000), but a higher proportion than that in Shanghai (no new case detected out of 44,000 people tested). ${ }^{6,7,22}$ We also found a higher proportion of repeat positives than Wuhan (1.3\% vs. $0.3 \%$, suggesting that mass testing performed during an earlier fadeout phase was perhaps more likely to detect cases of long-term intermittent shedding. ${ }^{6}$ Such findings indicated that the prevalence of infection may be overestimated in mass testing exercises if these types of cases are not accounted for. $^{23,24}$

The Hong Kong UCTP had a lower participation proportion (25\%) than the program in Wuhan (93\%), likely due to its voluntary nature. ${ }^{6}$ We found that participation was lowest among people 20 to 29 years of age, which was consistent with our survey results including a lower intention to participate and higher extent of concerns about the UCTP in this age group. Our survey results suggest that strengthening public 
communications about the safety and importance of mass testing may help address the common concerns and increase participation in future community-wide testing programmes.

Our study has several limitations. First, our reconstructed transmission chains were based on manual contact tracing, and links with other existing clusters might have been missed. Future genetic analysis could improve our understanding of the utilities of the UCTP and existing surveillance. Second, our telephone survey was conducted before the UCTP was launched, and therefore not able to directly assess the differences in the concerns between UCTP participants and non-participants. However, we believe that our survey results reflected the perceptions and concerns in the general adult population in Hong Kong at the time of the UCTP. Third, in our analysis we assumed that the proportion of detection after infections in each age group was stable during the third wave, which might have been affected by the evolving eligibility for testing in the existing surveillance in Hong Kong. However, most of the changes during our study period were largely reflected an expansion of the eligible population by including more high-risk groups, which only consisted of $0.5 \%$ of all COVID-19 local cases confirmed between 1 July to 30 September 2020.

Our study empirically revealed the utilities of population-wide mass testing in detecting unrecognised COVID-19 transmission on top of the existing proactive surveillance in the fadeout phase, especially for

detecting infected individuals without an epidemiologic link with other confirmed cases. Improving public communication about the safety and value of testing could help to enhance participation, particularly among young adults. We inferred that about 1 in 4 infections was detected by the existing clinical diagnosis and public health surveillance in Hong Kong between July and September 2020, with infections in children and older adults more likely to be missed.

\section{Declarations}

\section{ACKNOWLEDGMENTS}

We thank the Department of Health and Hospital Authority of the Food and Health Bureau of the Government of Hong Kong for conducting the outbreak investigation and providing the data for the analysis. This project was supported by the Health and Medical Research Fund, Food and Health Bureau, Government of the Hong Kong Special Administrative Region (grant no. COVID190118).

\section{AUTHOR CONTRIBUTIONS}

All authors meet the ICMJE criteria for authorship. BY, TKT, PW, BJC and GML conceived the study. BY, $H G, E H Y L, Y L, F H, D C A$ and JYW conducted the data analyses. BY, TKT, HG, EHYL, PW, BJC and GML interpreted the results. JX, QL and BJC conceived and performed the telephone surveys. BY wrote the first draft of the manuscript, and all authors provided critical review and revision of the text and approved the final version. 
BJC consults for Roche, Sanofi Pasteur, GSK and Moderna. The authors report no other potential conflicts of interest.

\section{References}

1. Real-time dashboard. https://covid19.sph.hku.hk/ (accessed March 3, 2021).

2. Wu P, Tsang T, Wong J, et al. Suppressing COVID-19 Transmission in Hong Kong: An Observational Study of the First Four Months. SSRN Electron J 2020; published online June 9. DOI:10.21203/rs.3.rs$34047 / \mathrm{v} 1$.

3. Cowling BJ, Ali ST, Ng TWY, et al. Impact assessment of non-pharmaceutical interventions against coronavirus disease 2019 and influenza in Hong Kong: an observational study. Lancet Public Heal 2020; 5: e279-88.

4. Leung GM, Cowling BJ, Wu JT. From a Sprint to a Marathon in Hong Kong. N Engl J Med 2020; 382: e45.

5. Kwok KO, Li KK, Chan HHH, et al. Community Responses during Early Phase of COVID-19 Epidemic, Hong Kong. Emerg Infect Dis 2020; 26: 1575-9.

6. Cao S, Gan Y, Wang C, et al. Post-lockdown SARS-CoV-2 nucleic acid screening in nearly ten million residents of Wuhan, China. Nat Commun 2020; 11. DOI:10.1038/s41467-020-19802-w.

7. Li Z, Liu F, Cui J, et al. Comprehensive large-scale nucleic acid-testing strategies support China's sustained containment of COVID-19. Nat Med 2021;: 1-2.

8. Xing Y, Wong GWK, Ni W, Hu X, Xing Q. Rapid Response to an Outbreak in Qingdao, China. N Eng/ J Med 2020; 383: e129.

9. news.gov.hk. https://www.news.gov.hk/eng/index.html (accessed Jan 13, 2021).

10. Yang B, Wu P, Y Lau EH, et al. Changing disparities in COVID-19 burden in the ethnically homogeneous population of Hong Kong through pandemic waves: an observational study. Clin Infect Dis 2021. DOI:10.1093/cid/ciab002.

11. 2016 Population By-census. https://www.bycensus2016.gov.hk/en/index.html (accessed Sept 7, 2020).

12. Adam DC, Wu P, Wong JY, et al. Clustering and superspreading potential of SARS-CoV-2 infections in Hong Kong. Nat Med 2020; 26: 1714-9.

13. He X, Lau EHY, Wu P, et al. Temporal dynamics in viral shedding and transmissibility of COVID-19. Nat Med 2020; 26: 672-5.

14. Hellewell J, Russell TW, Beale R, et al. Estimating the effectiveness of routine asymptomatic PCR testing at different frequencies for the detection of SARS-CoV-2 infections. medRxiv 2020. DOI:10.1101/2020.11.24.20229948.

15. O'Driscoll M, Dos Santos GR, Wang L, et al. Age-specific mortality and immunity patterns of SARSCoV-2. Nature 2020. DOI:10.1038/s41586-020-2918-0. 
16. Kucharski AJ, Klepac P, Conlan AJK, et al. Effectiveness of isolation, testing, contact tracing, and physical distancing on reducing transmission of SARS-CoV-2 in different settings: a mathematical modelling study. Lancet Infect Dis 2020; 20: 1151-60.

17. Du Z, Pandey A, Bai Y, et al. Comparative cost-effectiveness of SARS-CoV-2 testing strategies in the USA: a modelling study. Lancet Public Heal 2021; 6: 184-91.

18. Riley $\mathrm{S}$, Walters $\mathrm{CE}$, Wang $\mathrm{H}$, et al. REACT-1 round 7 updated report: Regional heterogeneity in changes in prevalence of SARS-CoV-2 infection during the second national COVID-19 lockdown in England. medRxiv 2020. DOI:10.1101/2020.12.15.20248244.

19. Brauner JM, Mindermann S, Sharma M, et al. Inferring the effectiveness of government interventions against COVID-19. Science 2020;: eabd9338.

20. Yang B, Huang AT, Garcia-Carreras B, et al. Effect of specific non-pharmaceutical intervention policies on SARS-CoV-2 transmission in the counties of the United States. medRxiv 2020.

21. Lessler J, Grabowski MK, Grantz KH, et al. Household COVID-19 risk and in-person schooling. Science 2021;: eabh2939.

22. Shanghai Municipal Health Commission. http://wsjkw.sh.gov.cn/ (accessed April 20, 2021).

23. AlJishi JM, Al-Tawfiq JA. Intermittent viral shedding in respiratory samples of patients with SARSCoV-2: observational analysis with infection control implications. J Hosp Infect 2021; 107: 98-100.

24. Mattiuzzi C, Henry BM, Sanchis-Gomar F, Lippi G. Sars-cov-2 recurrent rna positivity after recovering from coronavirus disease 2019 (COVID-19): A meta-analysis. Acta Biomed 2020; 91: 1-7.

\section{Tables}

Table 1. Characteristics of samples tested in the universal community test programme (UCTP) in Hong Kong. 


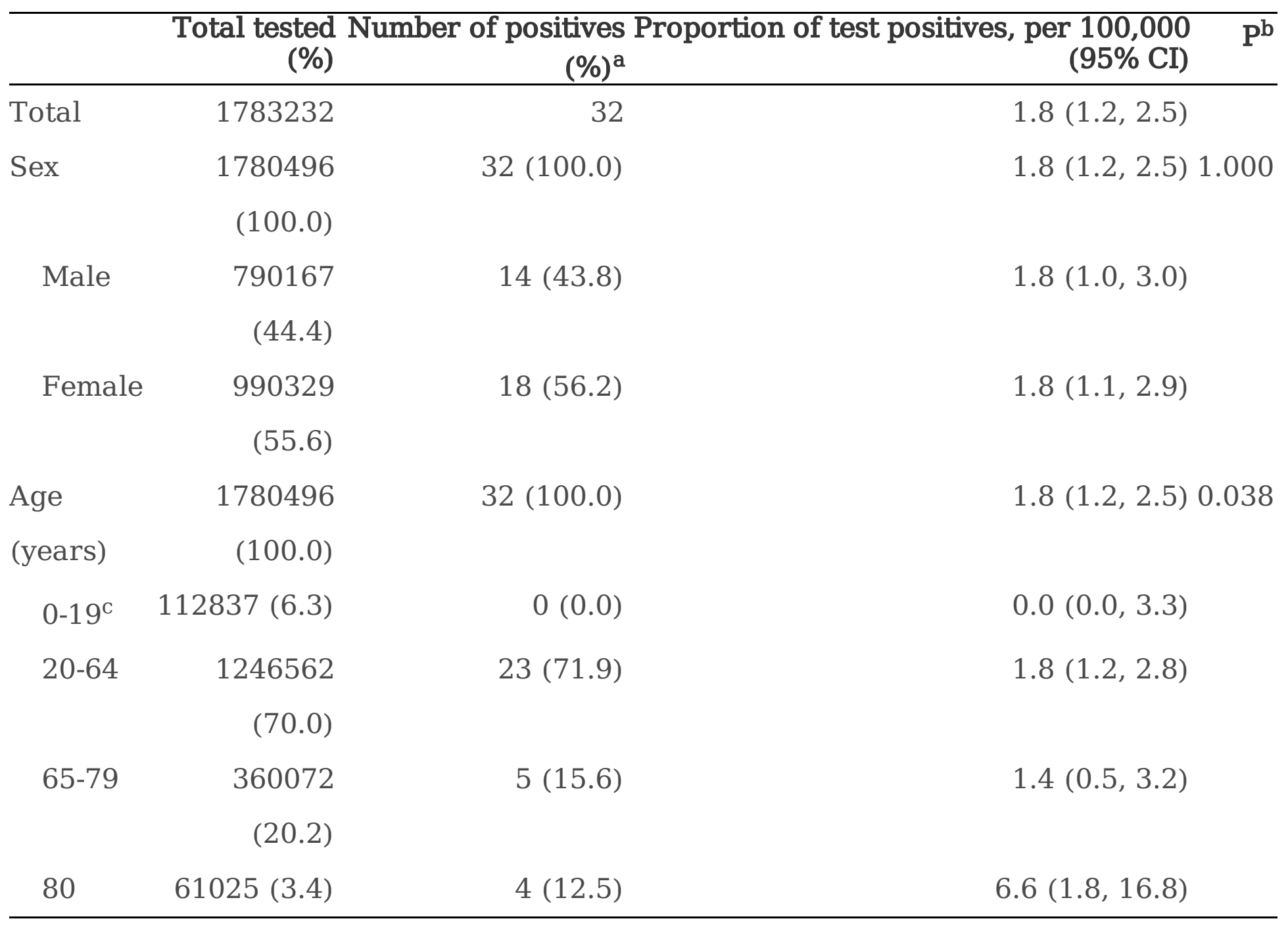

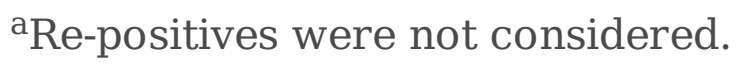

${ }^{\mathrm{b}}$ Chi-squared test or Fisher exact test were performed.

${ }^{\mathrm{C}} 265$ individuals aged 5 years or younger also participated the UCTP, though they might not be eligible.

\section{Figures}




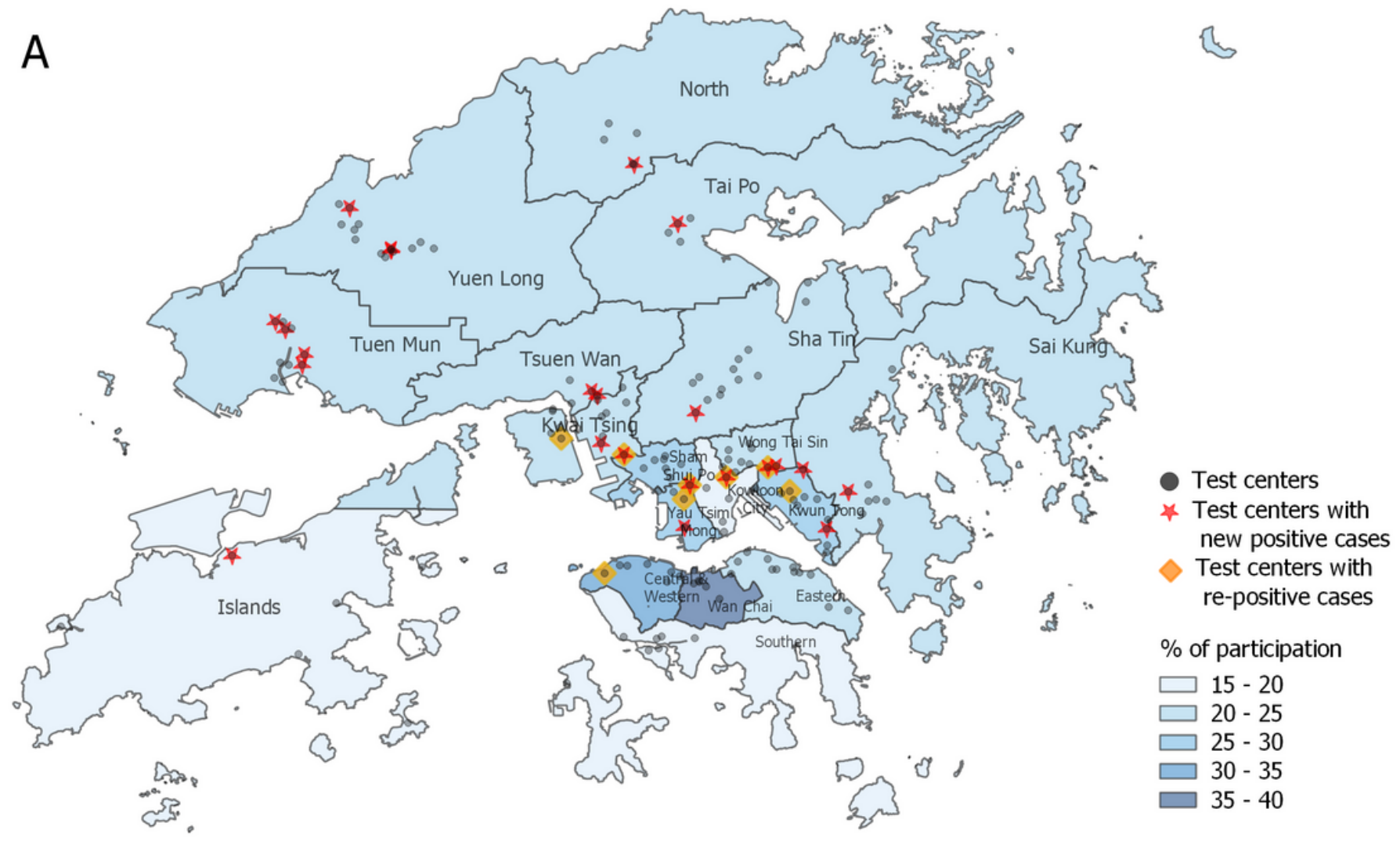

B

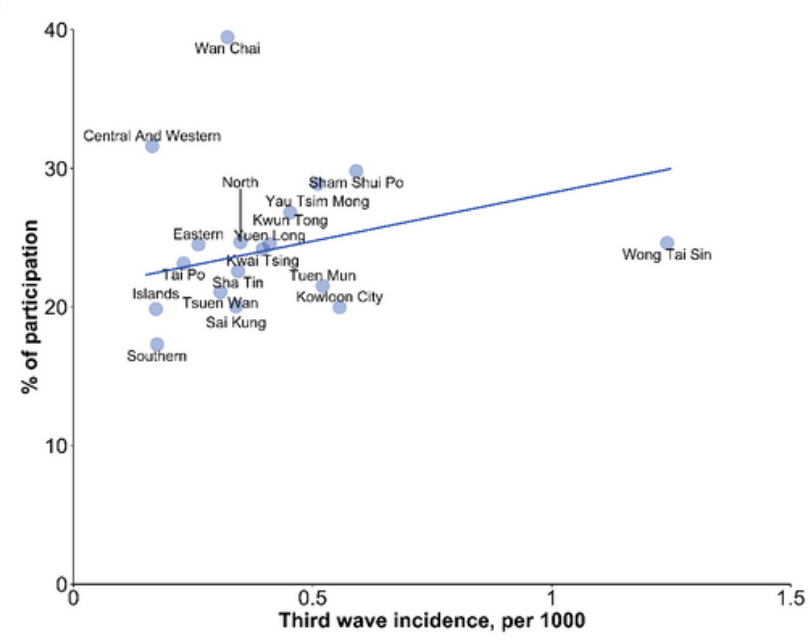

C

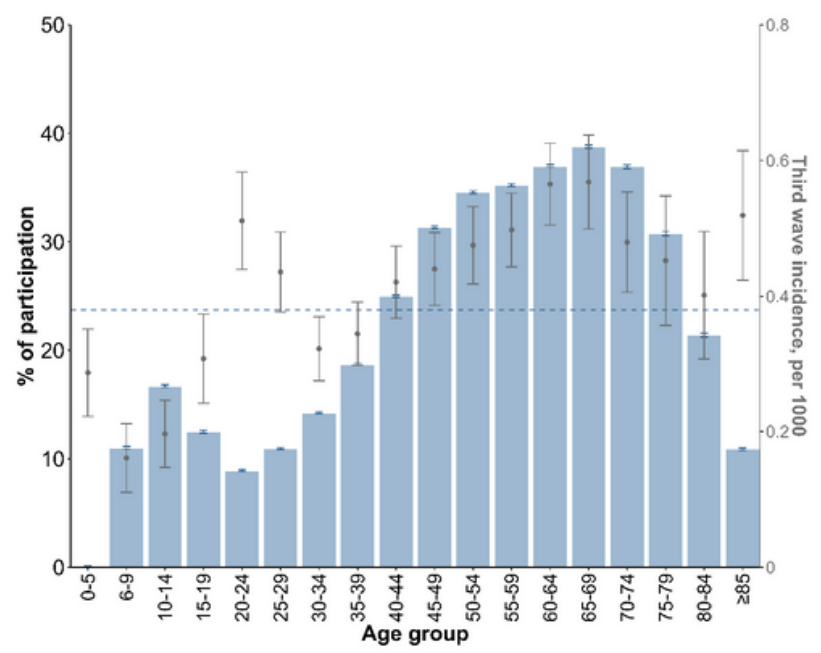

\section{Figure 1}

Participation in the universal community testing programme (UCTP) in Hong Kong during 1 to 14 September 2020. (A) Locations of all community testing centres and the centres with positive results. Background colours indicate the proportion of participation for 18 districts. (B) Association between the proportion of participation and the preceding incidence by district. The solid line indicates the best fitting linear regression model with data excluding Wan Chai and Central and Western. (C) Association between the proportion of participation (bars) and the preceding incidence (dots and line segments) by age group. 
Blue dashed horizontal line indicates the average proportion of participation. Note: The designations employed and the presentation of the material on this map do not imply the expression of any opinion whatsoever on the part of Research Square concerning the legal status of any country, territory, city or area or of its authorities, or concerning the delimitation of its frontiers or boundaries. This map has been provided by the authors.

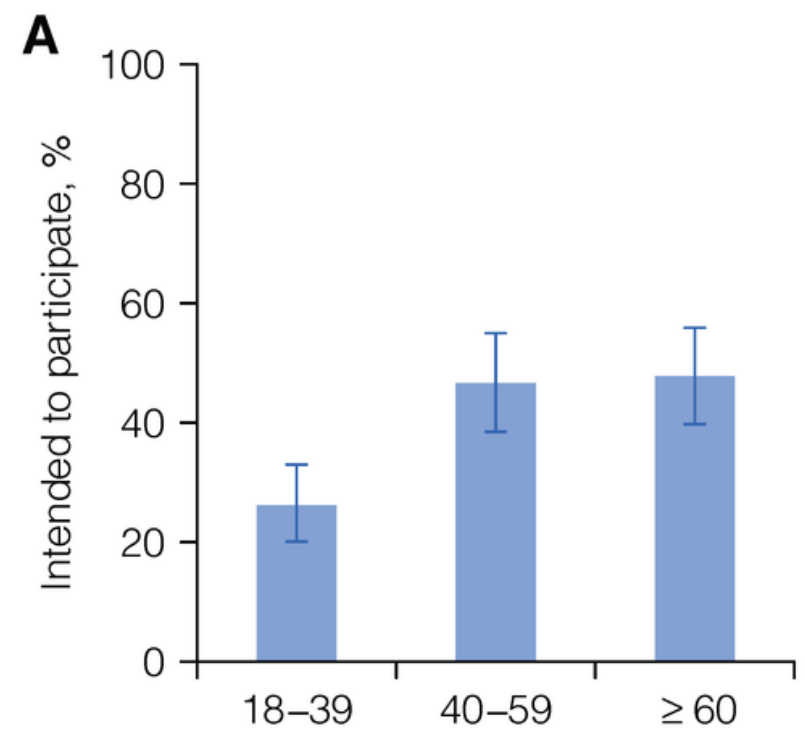

Age group

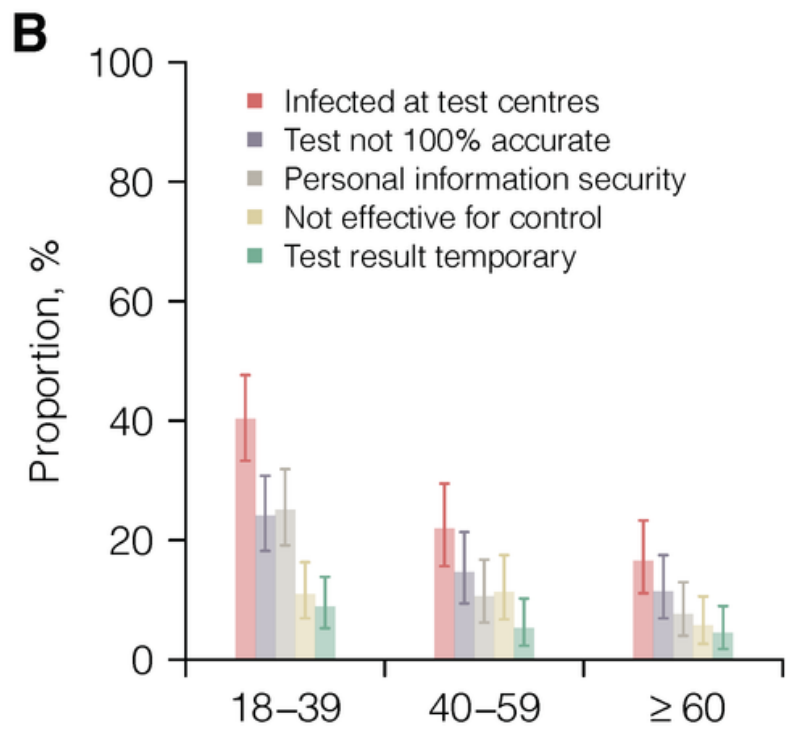

Age group

\section{Figure 2}

Intention to participate (A) and major concerns (B) about participating the universal community testing programme (UCTP) in the telephone survey in Hong Kong.

A

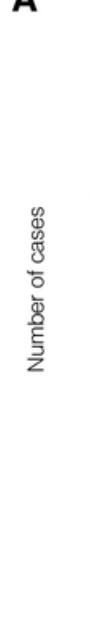

1507 . Clinical diagnosis and
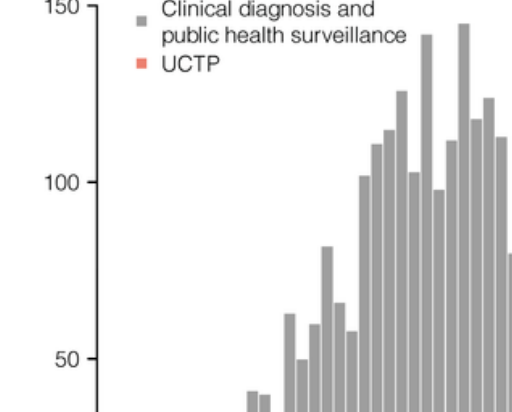

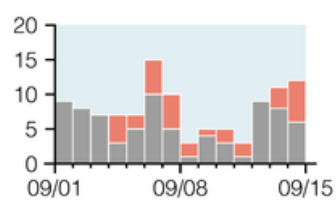

.
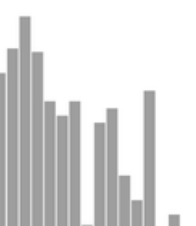
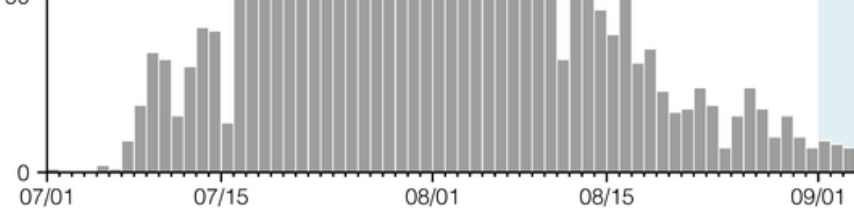

Date of report

B

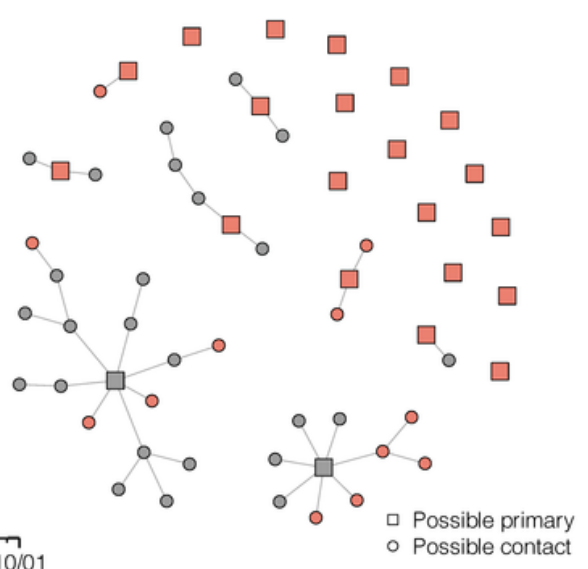

Figure 3 
Comparison of COVID-19 cases detected from the universal community testing programme (UCTP) and existing clinical diagnosis and public health surveillance in Hong Kong in July-September 2021. (A) Daily numbers of laboratory-confirmed COVID-19 cases detected by the existing surveillance and the UCTP in the third pandemic wave $(n=3,370)$. Imported cases were excluded. (B) The 32 newly identified COVID-19 cases by the UCTP and the reconstructed transmission chains linked to some of the cases.

A

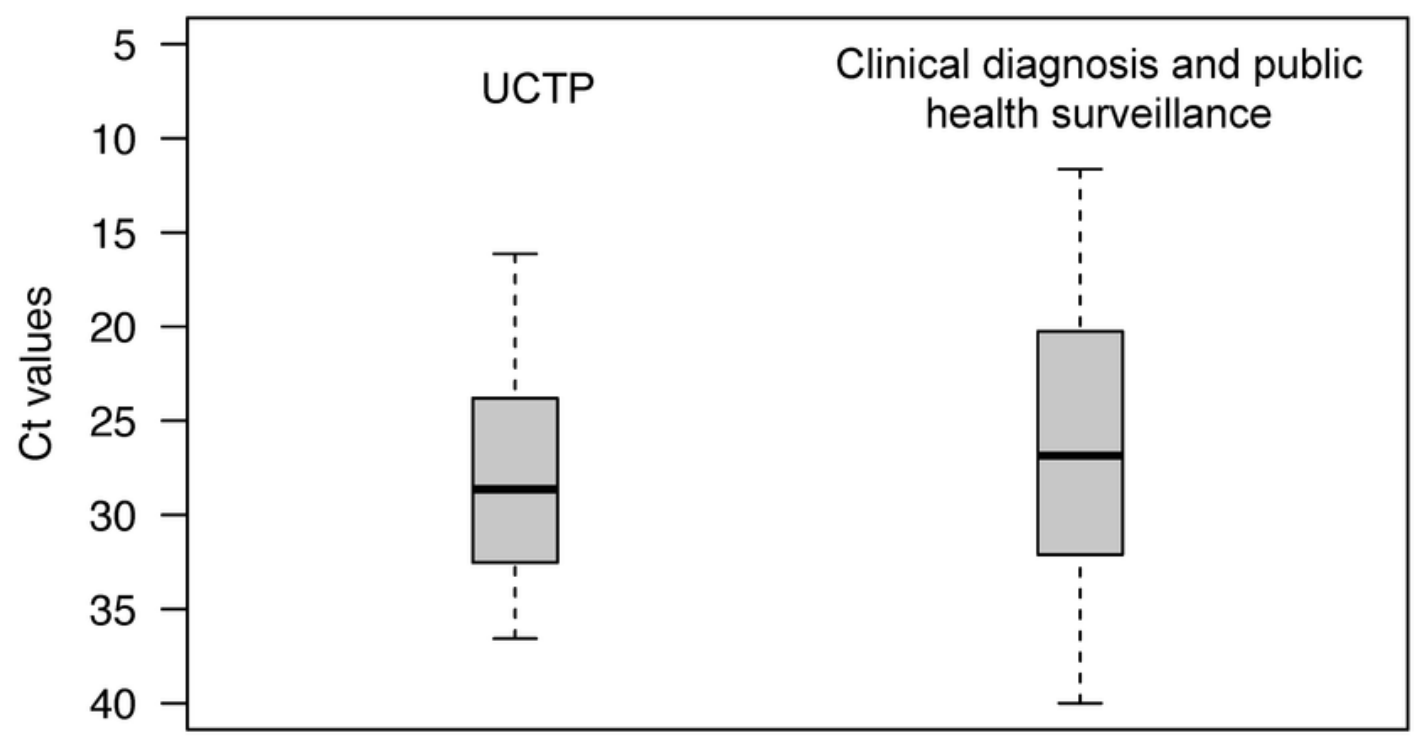

B

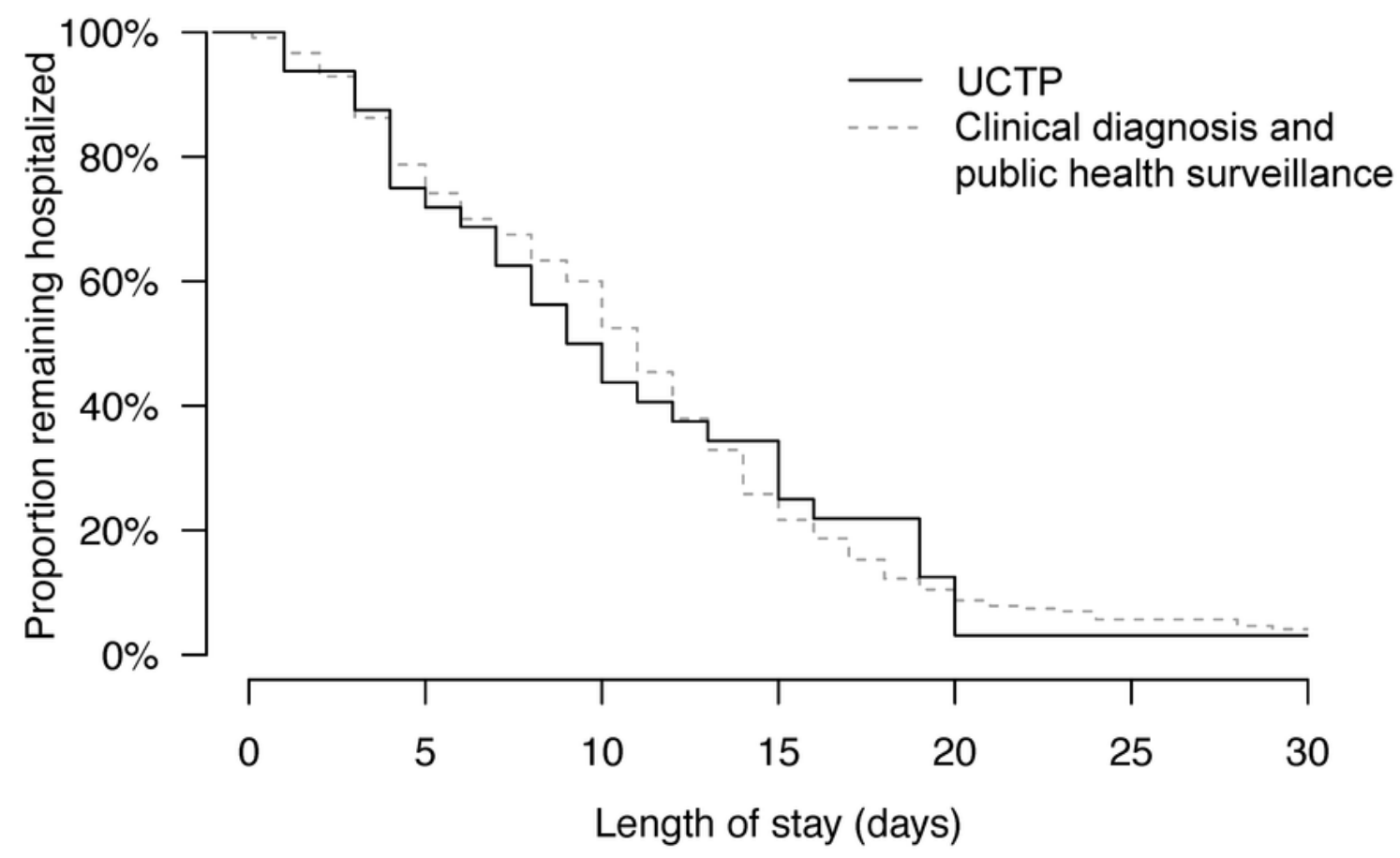

Figure 4 
Comparison of the cycle threshold (Ct) value (A) and the length of hospital stay (B) between COVID-19 cases detected by the existing clinical diagnosis and public health surveillance and the UCTP in the third pandemic wave in Hong Kong.

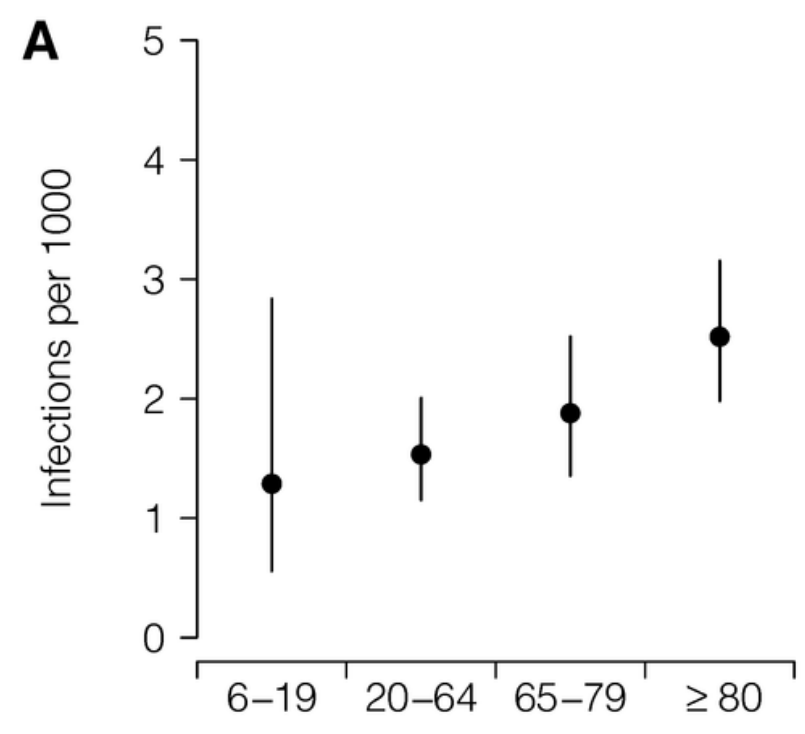

Age group

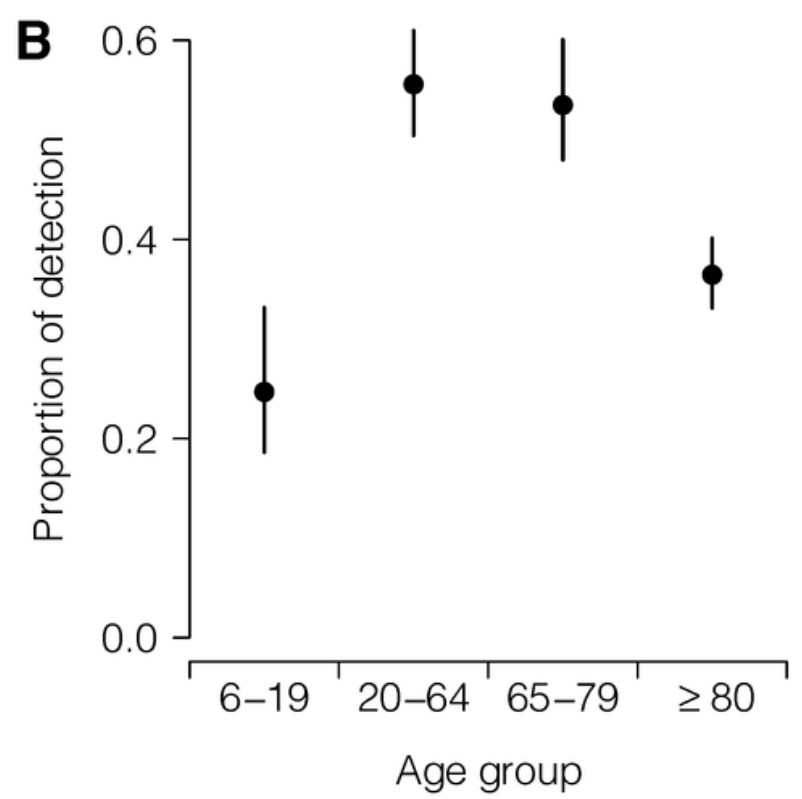

Age group

\section{Figure 5}

Estimated age-specific cumulative incidence $(A)$ and proportion of detection by existing clinical and public health surveillance (B) of COVID-19 during the third pandemic wave in Hong Kong.

\section{Supplementary Files}

This is a list of supplementary files associated with this preprint. Click to download.

- Researchincontext.docx

- Appendix.docx

- figures1.png

- figures2.png

- figures3.png

- SupplementaryTables.docx 